\title{
Trends in teaching lens design
}

Jose Sasian

Jose M. Sasian, "Trends in teaching lens design," Proc. SPIE 4588, Seventh International Conference on Education and Training in Optics and Photonics, (28 May 2002); doi: 10.1117/12.468682

SPIE Event: Education and Training in Optics and Photonics 2001, 2001, Singapore, Singapore 


\title{
Trends in Teaching Lens Design
}

\author{
Jose M. Sasian \\ Optical Sciences Center \\ University of Arizona \\ Tucson, Arizona 85721 \\ USA
}

\begin{abstract}
Lens design is a widely spread field of optical engineering and virtually all opticaldevices that are manufactured involve some lens design. Lens design is considered at the University of Arizona as an important discipline and courses are offered at the Master's and Ph. D. levels. Although lens design involves simple geometrical concepts, the grasp by students of this discipline is not easy. It takes time, repetition of concepts, and homework exercises for students to grasp and then master the subject. Therefore, we have carefully chosen our approach to teaching lens design so that students, after two courses are prepared to do useful work at the industry level. In this paper we present our approach to teaching lens design.
\end{abstract}

\section{TEACHING LENS DESIGN BACKGROUND}

The Optical Sciences Center at the University of Arizona is one of a few academic centers in the world that offers comprehensive training in lens design. Considerable attention is given to how lens design is taught, since students must grasp the fundamentals and acquire practical skills in a sixteen-week course. The course emphasizes imaging and aberration theory, software mastery, and actual lens design experience as important milestones toward acquiring the skill of lens design. Students are expected to develop an understanding of lens design fundamentals and to comprehend in detail how classical lenses work, from the landscape lens to the double Gauss lens. Several lens design software packages are available to students so that they become exposed to actual professional tools. By the end of the course, students are expected to analyze, optimize, and tolerance simple to medium complexity lens systems. In an advanced lens design seminar, students focus on the complete design of typical industry lens systems for micro-lithography, photography, display systems, microscopy, and zoom lenses.

The introductory course has evolved from material in the books Lens Design Fundamentals by Rudolf Kingslake ${ }^{1}$ and The Art and Science of Optical Design by Robert R. Shannon. ${ }^{2}$ A didactic and historical methodology to teaching lens design is outlined in R. Kingslake's book. In this book the working fundamentals and lens design principles are clearly shown. Several classical lenses are discussed, and the material is outlined in a logical and pedagogical manner. The book by R. Shannon points out many modern aspects of lens design and the use of optical design software. It describes clearly how to carry out the design of a lens, from initial layout to the final analysis and tolerance 
evaluation. Both of these books serve as important references for our introductory course in lens design. Some material is added to help students better understand lens design. For example, null corrector design is discussed to show how spherical aberration can be controlled. Optical design with diffracted lenses is also taught.

\section{INTRODUCTORY LENS DESIGN COURSE}

The course starts with a discussion of imaging and the collinear transformation. First order optics and wave aberrations, fourth order and sixth order, are reviewed. Then different techniques are shown in detail to control or correct each aberration. The wave aberration function, the sine condition, the Coddington equations, and the algorithm for sequential ray tracing is discussed. This material serves as the foundation of the course. Then the design and inner workings of the classical lenses is discussed. These being the Landscape lens, Periscope, Chevalier, Petzval Portrait, New Achromat, Rapid Rectilinear, Protar, Dagor, Cooke triplet, double Gauss, and Tessar lens. The teaching philosophy is to shown students how these lens designs of the $19^{\text {th }}$ century were designed and improved for photography. Live demonstrations with a laptop computer and a LCD projector are very valuable in showing how lens design is done. Historical notes are added to make the subject more interesting. Computer optimization, image evaluation, and tolerancing are also discussed and nine homework assignments complete the course. There is a midterm examination and a final report where students summarize the course contents to ease reviewing the course at a future time. A web site is available to students so they can unload class notes, homework assignments, and other pertinent material.

\section{ADVANCED LENS DESIGN SEMINAR}

The advanced lens design course is conducted as a seminar. Students are asked to select a lens design project and complete it from conception to reporting. Throughout the semester, students discuss their project progress, and suggestions are given as they report in front of other students. The teaching philosophy in the seminar is to expose students to typical industry lens design problems, and to have the students solve such problems, as they would be expected to do in industry. In addition, some advanced topics are discussed such as off-axis and unsymmetrical systems, eyepiece design, microscope objectives, and zoom lens design theory.

\section{CONCLUSIONS}

The majority of the students enjoy the introductory course, and work hard doing homework to learn the material. Students who have a strong interest in lens design become fully satisfied with the approach and contents of the courses. Students who are interested in optical engineering benefit from taking the introductory lens design course and the advanced seminar. The teaching approach in these courses has been carefully chosen to maximize the student's learning of the skill of lens design.

\section{ACKNOWLEDGMENT}

I would like to thank Professor Robert R. Shannon for sharing his teaching experience. ${ }^{3}$ 


\section{REFERENCES}

1. Rudolf Kingslake, Lens Design Fundamentals, Academic Press, San Diego,1978.

2. Robert R. Shannon, The art and Science of Optical Design, University Press, Cambridge, 1997.

3. Robert R. Shannon, 'Teaching Lens Design,' Optical Engineering 32 (8), 1722-1725, 1990. 